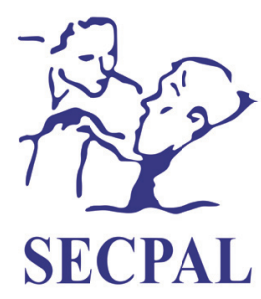

Medicina Paliativa

www.medicinapaliativa.es

ORIGINAL

\title{
Nivel de conocimientos en cuidados paliativos de las enfermeras; un estudio descriptivo en el Área de Salud VII de la Región de Murcia
}

\author{
Alberto Hiciano Guillermo ${ }^{1,2^{*}}$, Marta Jover Aguilar ${ }^{2}$ y Laura Martínez-Alarcón ${ }^{2-4}$ \\ ${ }^{1}$ Hospital General Universitario Reina Sofía. Murcia, España. ${ }^{2}$ Instituto Murciano de Investigación Biosanitaria

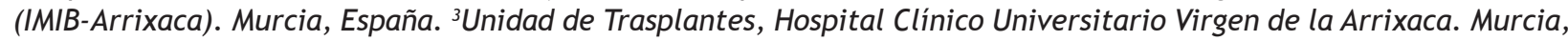 \\ España. ${ }^{4}$ Facultad de Enfermería, Universidad de Murcia. Murcia, España
}

Recibido el 21 de noviembre de 2019

Aceptado el 12 de abril de 2020

Se han presentado datos parciales en el V Congreso Nacional de Jóvenes Investigadores en enfermería, celebrado en la Universidad de Murcia, del 16 al 17 de octubre de 2019.

\author{
PALABRAS CLAVE \\ Cuidados paliativos, \\ enfermería, \\ conocimiento, \\ percepción, encuestas \\ y cuestionarios.
}

\begin{abstract}
Resumen
Introducción: Las enfermeras en ocasiones no se sienten lo suficientemente preparadas para proporcionar unos cuidados paliativos de calidad. El objetivo del presente estudio fue evaluar el nivel de conocimientos que poseen las enfermeras sobre conceptos básicos de cuidados paliativos y las variables que se relacionan con dicho nivel de conocimientos.

Material y métodos: Estudio descriptivo transversal realizado en Atención Primaria y Hospitalaria a 338 enfermeras del Área VII de la Región de Murcia entre marzo y mayo de 2018. Se empleó el cuestionario validado "Palliative Care Quiz for Nursing”, autocumplimentado y anónimo. Se llevó a cabo un análisis descriptivo de las variables y un análisis de correlación entre las variables con la puntuación media obtenida.

Resultados: Grado de cumplimentación: 82,25\% ( $\mathrm{n}=278)$; siendo 226 de las participantes mujeres (edad media de 43,6 \pm 10,6 años). El 65,7 \% obtuvo un nivel bajo de conocimientos en cuidados paliativos, con una puntuación media de 10,35 \pm 2,67 de aciertos sobre un total de 20 cuestiones. Se identificaron diferencias significativas de la media de aciertos en función de tener formación en cuidados paliativos $(p<0,007)$, las horas de formación $(p<0,008)$, tener experiencia en cuidados paliativos $(p<0,001)$ y el sentirse profesionalmente capacitado para proporcionar cuidados paliativos ( $p<0,001$ ). El 89,6\% consideraba insuficiente la formación de enfermería en cuidados paliativos y el $48,2 \%$ no se sentía lo suficientemente capacitada para proporcionar cuidados paliativos.
\end{abstract}

*Autor para correspondencia:

Alberto Hiciano Guillermo

Hospital General Universitario Reina Sofía. Avda. Intendente Jorge Palacios, nº 1, 30003, Murcia, España

Correo electrónico: albertohicianoguillermo@hotmail.com

1134-248X/ ( 2020 Sociedad Española de Cuidados Paliativos. Publicado por Inspira Network. Todos los derechos reservados. 
Discusión: La mayoría de las enfermeras del Área de Salud VII tenían un nivel bajo de conocimientos en cuidados paliativos. La realización de cursos de formación podría aumentar sus conocimientos, además de incrementar la calidad de los cuidados proporcionados.

\section{KEYWORDS}

Palliative care, nursing, knowledge, perception, surveys and questionnaires.

Hiciano Guillermo A, Jover Aguilar M, Martínez-Alarcón L. Nivel de conocimientos en cuidados paliativos de las enfermeras; un estudio descriptivo en el Área de Salud VII de la Región de Murcia. Med Paliat. 2020;27(2):79-87

\section{Introducción}

En España, no fue hasta finales del siglo xx cuando se desarrollan las primeras unidades de cuidados paliativos $(C P)^{1-3}$. Los pacientes en la fase final de la vida tienen múltiples necesidades que deben ser satisfechas y el objetivo de los profesionales (enfermeras, psicólogos, trabajadores sociales, médicos, etc.) que les atienden no es otro que procurar unos cuidados que permitan, tanto a los pacientes como a sus familias, alcanzar la mejor calidad de vida posible ${ }^{1-4}$. Dentro del equipo multidisciplinar son las enfermeras quienes juegan un papel clave al ser las profesionales que adquieren una mayor proximidad y pasan más tiempo con los pacientes al procurar unos cuidados directos, siendo el enlace entre el binomio paciente-familia y el resto del equipo ${ }^{1,3,4}$.

Actualmente, en el ámbito asistencial de nuestro país conviven enfermeras procedentes de tres planes de estudios distintos: ayudante técnico sanitario, diplomada en enfermería y graduada en enfermería, finalmente ${ }^{1}$. La formación en CP se ha ido incorporando progresivamente, siendo inexistente en los primeros planes de estudios, optativos en la diplomatura y obligatorios en el grado ${ }^{1,3}$. La mayor parte de enfermeras que trabajan actualmente en los distintos servicios de salud del país proceden de los dos primeros planes de estudios, lo que hace que la mayoría haya adquirido los conocimientos que tienen en cuidados paliativos con la experiencia o mediante formación continuada ${ }^{1,5}$. La Asociación Española de Enfermería en Cuidados Paliativos (AECPAL) divide en tres el nivel de conocimientos: básico, intermedio y avanzado; siendo el nivel básico el que todas las enfermeras deberían adquirir durante su etapa universitaria o con formación posgrado ${ }^{5}$. La formación de los profesionales juega un papel fundamental en los cuidados que se proporcionan 1,3,4. La falta de formación específica en CP y la diferencia de los contenidos impartidos a nivel pregrado pueden jugar un factor determinante que no solo afectaría a la calidad de los cuidados proporcionados, sino que también aumentaría el riesgo de aparición del "síndrome de burnout" en las enfermeras ${ }^{3,6,7}$. Gran parte de la bibliografía señalada realizada en países como Estados Unidos ${ }^{8,9}$, Austria $^{7}$, España ${ }^{10-12}$, 
Noruega ${ }^{13}$ o India ${ }^{14}$ han obtenido como conclusión que las enfermeras tienen un nivel bajo o medio de conocimientos en CP. Las escasas investigaciones y el escaso tamaño muestral de los estudios consultados en nuestro país hacen que no se haya analizado con detalle el nivel de conocimientos de las enfermeras, por lo que la realización de un estudio con un mayor tamaño muestral y que valore el nivel de conocimientos en ambos entornos asistenciales (Atención Hospitalaria y Atención Primaria) podría ayudar a clarificar esta cuestión ${ }^{10-12}$.

Por tanto, el objetivo principal de este estudio es evaluar el nivel de conocimientos que poseen las enfermeras sobre conceptos básicos de CP y determinar las variables que se relacionan con dicho nivel de conocimientos de manera significativa.

\section{Material y métodos}

\section{Tipo de estudio}

Se llevó a cabo un estudio descriptivo transversal realizado en Atención Primaria y Hospitalaria del Área de Salud VII de la Región de Murcia desde marzo hasta mayo de 2018, ambos inclusive.

\section{Población a estudio}

La población diana de este estudio fueron las enfermeras del Área de Salud VII (Murcia/Este), una de las 9 áreas de salud en la que se encuentra dividida la Región de Murcia. Dentro de esta área encontramos 12 zonas básicas de salud, donde pueden identificarse 12 centros de salud y 17 consultorios médicos. Además, encontramos el hospital de referencia: el Hospital General Universitario Reina Sofía. Los criterios de inclusión tenidos en cuenta fueron los siguientes: ser enfermera (independientemente del plan de estudios realizado); trabajar en el Área de Salud VII en el momento del estudio (en Atención Primaria o Atención Hospitalaria); pertenecer a la Administración Pública, estando contratado como personal estatutario fijo o temporal (interino, eventual o sustituto), y querer participar voluntariamente en el estudio. Se excluyeron a las estudiantes de enfermería, a las enfermeras que trabajaban en centros privados y los cuestionarios incompletos.

\section{Tamaño muestral}

La población de enfermeras en el Área VII en diciembre de 2017 fue de 486; 374 trabajaban en Atención Hospitalaria y 112 en Atención Primaria. La población accesible fue de 338 participantes, obteniendo una muestra de 281 enfermeras. La tasa de reducción fue de 1,06 \%, debido a que estos cuestionarios fueron cumplimentados por estudiantes de enfermería; siendo la muestra final de 278 participantes.

\section{Variables}

La variable dependiente fue el nivel de conocimientos de las enfermeras relacionado con conceptos básicos de CP. Las variables independientes fueron un total de 18 cuestiones que recogían las variables sociodemográficas (sexo, edad, hijos, estado civil y entorno asistencial), las de formación (plan de estudios, universidad y campus, realización de la asignatura de $\mathrm{CP}$, formación en $\mathrm{CP}$, tipo de formación y horas de formación en CP) y las de experiencia (años de experiencia profesional, experiencia en $\mathrm{CP}$, contacto con pacientes terminales; utilización y/o conocimiento del Plan Integral de CP de la Región de Murcia, opinión acerca de la formación que reciben las enfermeras en $C P$ y la capacitación de las enfermeras para proporcionar (P).

La herramienta utilizada fue el cuestionario "Palliative Care Quiz for Nursing” (PCQN) validado al castellano en el año $2017^{12}$. Se trata de un cuestionario compuesto por 20 ítems con tres opciones de respuesta (Verdadero/Falso/ No lo sé). Los ítems se dividen en 3 dimensiones de los cuidados paliativos: "La filosofía y los principios de estos cuidados" (4 ítems: 1, 9, 12 y17), "El tratamiento del dolor y de otros síntomas" (13 ítems: 2, 3, 4, 6, 7, 8, 10, 13, 14, 15, 16, 18, 20) y “Aspectos psicosociales" (3 ítems: 5, 11, $19)^{14}$. Las respuestas de las participantes se puntuaron con 1 punto en el caso de indicar la opción correcta o con 0 puntos en el caso de que el participante indicara la opción incorrecta o indicara la opción "No lo sé”. La puntuación total oscilaba entre 0 puntos y un máximo de 20 puntos. El nivel de conocimientos se ha clasificado de la siguiente manera:

Nivel bajo de conocimientos: porcentaje de aciertos inferior al $60 \%$ (0-11 aciertos).

Nivel medio de conocimientos: porcentaje de aciertos entre el 60-80\% (12-16 aciertos).

- Nivel alto de conocimientos: porcentaje de aciertos superior al $80 \%$ (17-20 aciertos).

El cuestionario entregado constaba de las siguientes partes: carta de presentación, 18 cuestiones acerca de variables sociodemográficas, de formación y de experiencia sobre los CP, y el cuestionario PCQN. Este puede consultarse en el Anexo I.

\section{Reclutamiento}

La distribución de los cuestionarios y reclutamiento de las participantes se llevó a cabo entre los meses de marzo y mayo de 2018. Se le proporcionó a cada participante un cuestionario donde constaban las 18 cuestiones acerca de variables sociodemográficas y el cuestionario PCQN. Se administró de forma autocumplimentada y era de participación voluntaria, individual y anónima. En Atención Hospitalaria se distribuyó en 33 de los 37 servicios con los que cuenta el Hospital General Universitario Reina Sofía, entre los que se encuentran las unidades de hospitalización, consultas externas, servicio de urgencias, administración y del resto de servicios (diálisis, farmacia, radiodiagnóstico, quirófano, reanimación, unidad de cuidados intensivos, hospital de día médico y quirúrgico y el equipo de soporte de atención hospitalaria del Área VII). En Atención Primaria se realizó en los 12 centros de salud que tiene esta Área y en 15 de los 17 consultorios médicos. Además, se incluyó uno de los servicios de urgencias de Atención Primaria, al punto de atención continuada y al equipo de soporte de atención domiciliaria del Área de Salud VII. 
Para la entrega de los cuestionarios se siguió el siguiente plan de acción: el estudio comenzó a realizarse en los servicios del Hospital General Universitario Reina Sofía. Se explicó al supervisor de cada servicio la finalidad, los objetivos del estudio y se entregó un sobre cerrado donde depositar los cuestionarios. La entrega de los cuestionarios se realizó en días y turnos distintos, para que todas las enfermeras tuvieran la oportunidad de participar, explicando tanto de forma grupal como individual a las participantes la finalidad del estudio.

En el caso de Atención Primaria, se acudió al centro de salud donde se encontraba la coordinadora de enfermería o responsable de docencia para explicar la finalidad del estudio. Estas se encargaron de su distribución y recogida en las reuniones semanales que hacía el equipo de Atención Primaria. Una vez recogidos, los cuestionarios fueron guardados en un sobre cerrado hasta su recogida. En los consultorios médicos también se explicó de manera detallada el estudio y se designó a una enfermera responsable de guardar en un sobre cerrados los cuestionarios hasta su recogida.

A la hora del almacenamiento de los datos, los investigadores utilizaron técnicas de disociación y anonimización para que no fuera posible una relación entre el dato y el participante del estudio durante el manejo de los datos.

\section{Análisis estadístico}

Se realizó un análisis descriptivo de las variables sociodemográficas, las de formación, las de experiencia y de las puntuaciones obtenidas por las participantes en el cuestionario. Para estudiar la relación entre variables numéricas se utilizó el coeficiente de correlación de Pearson. Para estudiar la relación entre variables cuantitativas y cualitativas se utilizó la t de Student y ANOVA. Para la realización del análisis estadístico de los datos se creó una base de datos en el programa "Statistical Package for the Social Sciences" (SPSS) versión 24. Se ha considerado como estadísticamente significativa una $\mathrm{p}$ valor menor de 0,05 .

\section{Aspectos éticos y legales}

Para la realización de este estudio se obtuvo el visto bueno y la aprobación de la Dra. Frances Fothergill-Boubonnais (Universidad de Ottawa), de la Comisión de Ética de Investigación de la Universidad de Murcia (ID: 1751/2017) y de la Dirección de enfermería del Área de Salud VII de la Región de Murcia. El estudio se llevó a cabo en consonancia con el código ético de la OMS (Declaración de Helsinki). Los autores declaran que no existió conflicto de intereses para la realización de la investigación.

\section{Resultados}

De los 338 cuestionarios entregados, resultaron válidos 278 cuestionarios, por lo que el grado de cumplimentación de este estudio fue del $82,25 \%$.

\section{Características de las participantes}

La mayoría de los participantes eran mujeres (81,3\%), con una edad media de 43,6 $\pm 10,6$ años. La mayor parte de las enfermeras eran diplomadas en enfermería $(79,2 \%)$ y la media de experiencia profesional era de 18,57 \pm 10,49 años. Respecto al entorno asistencial donde trabajaban, el $59 \%$ de la muestra lo hacía en Atención Hospitalaria, mientras que el 41 \% de la muestra trabajaba en Atención Primaria. En relación con la formación en cuidados paliativos, el $49 \%$ de las enfermeras no había recibido formación específica. La distribución de las variables se especifica en la Tabla I.

\section{Nivel de conocimientos de conceptos básicos de cuidados paliativos}

La media de preguntas acertadas fue de 10,35 $\pm 2,67$ preguntas de las 20 del cuestionario, lo que supone un porcen-

Tabla I. Características de la muestra

\begin{tabular}{|c|c|}
\hline Variables & Distribución \\
\hline Sexo & $\begin{array}{l}\text { Hombre }(n=52): 18,7 \% \\
\text { Mujer }(n=226): 81,3 \%\end{array}$ \\
\hline Edad & $43,6 \pm 10,6$ años \\
\hline Hijos & $\begin{array}{l}\text { Sí }(n=194): 69,8 \% \\
\text { No }(n=83): 29,8 \% \\
\text { No sabe/No contesta }(n=1): 0,4 \%\end{array}$ \\
\hline Estado civil & $\begin{array}{l}\text { Soltero }(n=56): 20,1 \% \\
\text { Casado/En pareja }(n=181): 65,1 \% \\
\text { Separado/Divorciado/Viudo }(n=40): 14,4 \% \\
\text { No sabe/No contesta }(n=1): 0,4 \%\end{array}$ \\
\hline $\begin{array}{l}\text { Entorno } \\
\text { asistencial }\end{array}$ & $\begin{array}{l}\text { Atención Primaria }(n=114): 41 \% \\
\text { Atención Hospitalaria }(n=164): 59 \%\end{array}$ \\
\hline $\begin{array}{l}\text { Años de } \\
\text { experiencia } \\
\text { profesional }\end{array}$ & $18,57 \pm 10,49$ años \\
\hline $\begin{array}{l}\text { Plan de } \\
\text { estudios }\end{array}$ & $\begin{array}{l}\text { Ayudante Técnico Sanitario }(n=17): 6,1 \% \\
\text { Diplomatura en Enfermería }(n=220): 79,2 \% \\
\text { Grado en Enfermería }(n=41): 14,7 \%\end{array}$ \\
\hline $\begin{array}{l}\text { Universidad } \\
\text { y campus }\end{array}$ & $\begin{array}{l}\text { U. de Murcia - Espinardo }(n=165): 59,5 \% \\
\text { U. de Murcia - Lorca }(n=5): 1,8 \% \\
\text { U. de Murcia - Cartagena }(n=14): 5 \% \\
\text { U. Católica San Antonio - Los Jerónimos } \\
(n=41): 14,7 \% \\
\text { Otras }(n=49): 17,6 \% \\
\text { Desconocida }(n=4): 1,4 \%\end{array}$ \\
\hline $\begin{array}{l}\text { Formación } \\
\text { en cuidados } \\
\text { paliativos }\end{array}$ & $\begin{array}{l}\text { No recibió formación: } 49 \% \\
\text { Formación pregrado: } 21 \% \\
\text { Formación posgrado: } 17 \% \\
\text { Formación pre y posgrado: } 9 \% \\
\text { No especificado: } 4 \%\end{array}$ \\
\hline $\begin{array}{l}\text { Horas de } \\
\text { formación }\end{array}$ & $\begin{array}{l}\text { Inferior a } 80 \text { horas: } 29,1 \% \\
80-150 \text { horas: } 10,8 \% \\
\text { Más de } 15 \text { horas: } 5 \%\end{array}$ \\
\hline
\end{tabular}


taje medio de aciertos del 51,75 \% $\pm 21,15 \%$ de preguntas correctas. El mínimo de aciertos fue 0 y el máximo fue de 16 aciertos sobre las 20 cuestiones planteadas. Con respecto al nivel de conocimientos que poseían, el 65,7\% $(n=183)$ de la muestra obtuvo un nivel bajo y el $34,3 \%(n=95)$ obtuvo un nivel medio (porcentaje de aciertos entre el 60 y $80 \%$ ).

De las 3 dimensiones evaluadas por el cuestionario, la dimensión con un número mayor de aciertos fue la de "Filosofía y principios" (56,3\%), seguida de la dimensión “Control del dolor y otros síntomas” (53,65 \%). La dimensión “Aspectos psicosociales" fue la que menor porcentaje de aciertos obtuvo $(37,53 \%)$ (Tabla II).

Los conceptos con mayor porcentaje de aciertos estaban relacionados principalmente con la importancia de las terapias adyuvantes y con la prevención del estreñimiento al utilizar opioides (preguntas número 4 y 8 respectivamente); obteniendo en ambas un porcentaje de aciertos superior al $90 \%$. Por otro lado, conceptos como las necesidades de la familia obtuvieron un $24,5 \%$ de aciertos.

\section{Análisis bivariante}

Se analizaron las variables sociodemográficas, de formación y de experiencia relacionadas con la media de aciertos obtenida en el cuestionario PCQN. Con respecto a la uni- versidad y el campus de la Región de Murcia donde estudiaron las enfermeras, aparecieron diferencias significativas con la media de aciertos, donde la Universidad Católica de San Antonio de Murcia fue la universidad con mayor media de aciertos de la Región de Murcia (11,39 \pm 2,70 aciertos; $p<0,012$ ). Haber realizado formación en CP también mostró una diferencia significativa con la media de aciertos $(p<0,007)$, al igual que haber realizado más horas de formación $(p<0,008)$. En aquellas enfermeras que tenían experiencia profesional $(p<0,001)$ y que habían trabajado anteriormente en $C P(p<0,011)$ también se identificaron diferencias significativas con la media de aciertos obtenida, aumentando esta al aumentar los años de experiencia. Las participantes que se sentían lo suficientemente capacitadas para proporcionar CP también obtuvieron una mayor media de aciertos que las que no se sentían capacitadas, siendo esta diferencia estadísticamente significativa $(p<0,001)$. El nivel de correlación entre el resto de las variables y la media de aciertos obtenida se muestra en la Tabla III.

\section{Discusión}

El objetivo principal del presente estudio es evaluar el nivel de conocimientos de las enfermeras sobre conceptos básicos de CP, analizando las variables relacionadas

Tabla II. Nivel de conocimientos en conceptos básicos de cuidados paliativos

\begin{tabular}{lcc}
\hline \multicolumn{1}{c}{ Conceptos básicos } & Porcentaje medio de aciertos + DE & Porcentaje medio de errores + DE \\
\hline Filosofía y principios & $56,3 \% \pm 18,74$ & $43,7 \% \pm 18,74$ \\
\hline Control del dolor y otros síntomas & $53,65 \% \pm 22,57$ & $46,34 \% \pm 22,58$ \\
\hline Aspectos psicosociales & $37,53 \% \pm 17,13$ & $62,46 \% \pm 17,13$ \\
\hline
\end{tabular}

$D E$ : desviación estándar.

Tabla III. Correlación entre las variables y la media de aciertos

\begin{tabular}{ll}
\hline \multicolumn{1}{c}{ Variables } & $\mathrm{p}$-valor \\
\hline Sexo & $\mathrm{p}<0,83$ \\
\hline Edad & $\mathrm{p}<0,46$ \\
\hline Hijos & $\mathrm{p}<0,73$ \\
\hline Estado civil & $\mathrm{p}<0,35$ \\
\hline Nivel de atención sanitaria & $\mathrm{p}<0,46$ \\
\hline Años de experiencia & $\mathrm{p}<0,64$ \\
\hline Plan de estudios & $\mathrm{p}<0,07$ \\
\hline Resto de universidades y Escuelas & $\mathrm{p}<0,26$ \\
\hline de Enfermería & $\mathrm{p}<0,56$ \\
\hline Cursar asignatura de CP & $\mathrm{p}<0,16$ \\
\hline Plan Integral de CP de la Región de Murcia & $\mathrm{p}<0,34$ \\
\hline Opinión de formación de Enfermería en CP & \\
\hline CP: cuidados paliativos. &
\end{tabular}

con dicho nivel de conocimientos. Los resultados de este estudio indican que la mayor parte de enfermeras del Área de Salud VII de la Región de Murcia tenía un nivel bajo de conocimientos en cuidados paliativos. Estos resultados coinciden con múltiples estudios que también evaluaron el nivel de conocimientos de las enfermeras utilizando el mismo cuestionario ${ }^{15-19}$. Por otro lado, también se han identificado otras investigaciones en las que las enfermeras obtuvieron un nivel medio de conocimientos, 10,20-24. La menor media de aciertos del cuestionario se identificó en un estudio realizado en Irán ${ }^{16}$, obteniendo una media de 7,59 aciertos. La mayor media de aciertos se encontró en un estudio realizado en Estados Unidos, donde la media de aciertos fue de 13,52 aciertos $^{9}$. Los estudios que obtuvieron las menores medias de aciertos se realizaron en países donde los cuidados paliativos apenas están desarrollados e integrados, como Irán ${ }^{16}$ o República Democrática del Congo ${ }^{17}$, por lo que los factores educativos y culturales del país donde se realice el estudio podrían influir en la media de aciertos ${ }^{19}$. 
Con respecto a las tres dimensiones evaluadas por el cuestionario "Palliative Care Quiz for Nursing" (PCQN), encontramos que las dimensiones "Filosofía y principios" y "Manejo del dolor y otros síntomas" fueron las que obtuvieron mayor porcentaje de aciertos en varias investigaciones realizadas en países como Irán ${ }^{16}$, Jordania ${ }^{19}$ o España ${ }^{23}$, obteniendo un porcentaje de aciertos muy similar en ambas dimensiones ${ }^{12,16,19,23}$. Sin embargo, la dimensión “Aspectos psicosociales" obtuvo el menor porcentaje de aciertos, tanto en este como en otros estudios que la analizaban ${ }^{16-19,22-25}$. Este hecho puede hacer plantearnos si el aspecto psicosocial se trabaja realmente durante la formación de estos profesionales o esta solo se centra en la adquisición de conceptos meramente teóricos.

En cuanto a la formación recibida, aquellas enfermeras que tenían más horas de formación obtuvieron una mayor puntuación de aciertos; coincidiendo con gran parte de la bibliografía consultada ${ }^{10,15-18,20,21,23-25}$; excepto en uno de los estudios en los que esta relación no era significativa ${ }^{19}$. En relación con la experiencia profesional, se encontraron diferencias con la media de aciertos, coincidiendo con la versión original del cuestionario ${ }^{25}$ y estudios más recientes realizados en países como España ${ }^{10}$ o Bangkok ${ }^{24}$. Además, los años de experiencia profesional específicamente en CP mostraron una relación estadísticamente significativa y directamente proporcional con la media de aciertos. Sin embargo, estudios previos que evaluaron los años de experiencia profesional específicamente en $\mathrm{CP}$ no identificaron una relación con la media de aciertos ${ }^{10,15,17}$. En España, el último estudio realizado identificó que, cuantos más años de experiencia en CP, mayor media de aciertos ${ }^{23}$. Igualmente, la experiencia con pacientes terminales fue una variable que tanto en el presente estudio como en otros ha influido favorablemente en la media de aciertos $^{10,23,25}$, aunque otras investigaciones hayan encontrado lo contrario ${ }^{15,17,20}$. Todos estos resultados indican que el contacto con estos pacientes y la experiencia profesional son variables que influyen en el nivel de conocimientos ${ }^{10,23,25}$.

También se identificó una relación significativa de la media de aciertos en función de sentirse capacitado para proporcionar CP. De esta manera, las enfermeras que sí se sentían lo suficientemente capacitadas obtuvieron una mayor media de aciertos. Esta cuestión no se ha identificado en estudios anteriores, pero sí que se ha valorado en varios de ellos la actitud de las enfermeras hacia los cuidados paliativos ${ }^{16,17,19,20,26}$. Otros estudios que analizaban la actitud antes y después de recibir formación específica sobre CP reflejan que, al recibir dicha formación, su actitud hacia este tipo de cuidados mejoraba17,19,26.

Tal y como indican la Sociedad Española de Cuidados Paliativos (SECPAL), la actitud forma parte de los factores que comprenden las competencias de una profesional (conocimientos, actitudes y habilidades), existiendo entre estos una relación directamente proporcional ${ }^{5}$. Por ello, en futuros estudios, sería ideal la evaluación conjunta de los conocimientos, las actitudes y las habilidades de las enfermeras en cuidados paliativos ${ }^{21,26}$.

Los resultados obtenidos en este estudio podrían extrapolarse a la población de enfermeras del Área VII de la Región de Murcia, obteniendo la mayoría de ellas un nivel de conocimientos bajo en CP. Además, cerca del 50 \% de ellas no se sentían lo suficientemente capacitadas para proporcionar cuidados de tipo paliativo. En investigaciones futuras, podría realizarse una intervención a las enfermeras de nuestro país para evaluar si se produce un aumento de su nivel de conocimientos. La intervención no solo debería incluir aspectos teóricos sino trabajar las actitudes y habilidades de las enfermeras ${ }^{3}$. De esta manera, además de aumentar sus competencias en $\mathrm{CP}$, factores como el miedo o la incertidumbre podrían verse disminuidos, reduciendo así uno de los principales estresores de las enfermeras ${ }^{3}$. La realización de intervenciones de este tipo a enfermeras ha mostrado un aumento del nivel de conocimientos, habilidades y actitudes hacia los cuidados paliativos ${ }^{21,24,26}$.

Entre las limitaciones de este estudio, se valoró que pudiera existir un sesgo de selección debido a la distribución no probabilística de los cuestionarios y a la no inclusión de todos los servicios del Área de Salud. A pesar de esto, se incluyó a la mayor parte de servicios, centros y consultorios médicos de Atención Primaria y Hospitalaria del Área de Salud. De las 486 enfermeras que conformaban el Área de Salud VII, se accedió a 338 enfermeras. El 82,25\% de estas 338 enfermeras realizaron el cuestionario, por lo que el grado de cumplimentación fue alto $(n=278)$, además de obtener un número de participantes superior al tamaño mínimo muestral necesario para poder extrapolar los resultados al Área de Salud VII.

La otra limitación fue la no identificación de una única clasificación para categorizar el nivel de conocimientos según el porcentaje de aciertos. Una de estas clasificaciones resultaba totalmente subjetiva al utilizar como punto de corte la media de aciertos obtenida entre nivel bajo y alto. Otra de las clasificaciones consideraba un nivel de conocimientos alto a un porcentaje de aciertos superior al $75 \%$ y nivel bajo a un porcentaje inferior al $75 \%$, por lo que tampoco era posible identificar con esta el nivel medio de conocimientos. Por ello, se consultaron las clasificaciones realizadas por otras herramientas con la misma finalidad, empleando finalmente la del cuestionario "Emergency Nurses' Competency in Provision Palliative Care", que permitía identificar el nivel medio de conocimientos de una forma más objetiva que las anteriores ${ }^{27}$.

\section{Conclusiones}

Para concluir, indicar que la mayor parte de las enfermeras del Área VII de la Región de Murcia tenían un nivel bajo de conocimientos relacionados con conceptos básicos de cuidados paliativos. Haber realizado formación, tener experiencia profesional y sentirse capacitado para proporcionar CP fueron variables relacionadas con un mayor nivel de conocimientos.

\section{Agradecimientos}

A mi madre y a Javi por estar ahí siempre. A mi maestra y amiga, Laura Martínez-Alarcón y a Marta Jover por sus buenos consejos. A la Dra. Frances Fothergill-Boubonnais por facilitarnos la utilización del cuestionario. A Guadalupe Ruiz por ayudarme tanto con las bases de datos. A Elena Chover-Sierra por sus consejos constructivos sobre cómo abordar esta investigación. A Gloria Muñoz y a Fuensanta Sánchez y a todas las enfermeras y enfermeros que han hecho posible que este trabajo sea una realidad.

\section{Fuentes de financiación}

La presente investigación no ha recibido ayudas específicas provenientes de agencias del sector público, sector comercial o entidades sin ánimo de lucro. 


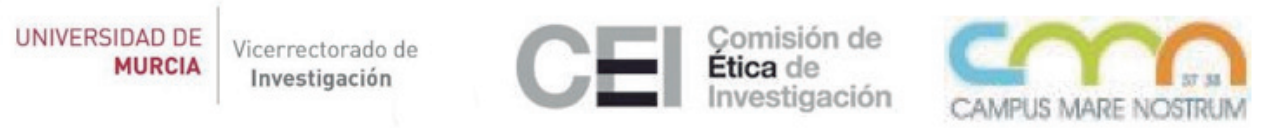

\title{
Anexo I. Hoja de información sobre el proyecto y cuestionario empleado.
}

\author{
HOJA DE INFORMACIÓN
}

Estimado Sr. o Sra.:

Le invitamos a participar en un estudio de investigación que tiene como título "Conocimientos del personal de Enfermería sobre los Cuidados Paliativos en el Área VII de la Región de Murcia”.

Objetivos y finalidad

El estudio pretende identificar el nivel de conocimientos que poseen los profesionales de Enfermería del Área VII de la Región de Murcia sobre conceptos básicos de los Cuidados Paliativos. El proyecto cuenta con el informe favorable de la Comisión de Ética de Investigación de la Universidad de Murcia.

Datos de los investigadores

Ante cualquier duda que pueda surgir en relación con su participación en la presente investigación, pueden dirigirse a la persona responsable de la misma:

- Nombres: Alberto Inocencio Hiciano Guillermo, Marta Jover Aguilar y Laura Martínez-Alarcón.

- Correo electrónico: albertohicianoguillermo@hotmail.com

Datos referentes al participante. Informar sobre lo siguiente:

- Se invita al personal de Enfermería a participar en nuestro estudio para conocer el nivel de conocimientos que tienen Estos sobre conceptos básicos de los Cuidados Paliativos.

- Naturaleza voluntaria de su participación. El participante es libre de participar, de modo que se puede negar a participar.

\section{Derechos de los participantes}

- Garantía de confidencialidad de la información obtenida, indicando la existencia del fichero, la finalidad de la recogida de los datos y destinatarios de la información, del carácter obligatorio o facultativo de las respuestas, de la posibilidad y lugar donde ejercer los derechos de acceso, rectificación, cancelación y oposición, de la identidad y dirección del responsable del fichero, el modo en que se manejarán las bases de datos y la identidad de las personas que tendrán acceso a los datos de carácter personal del sujeto fuente.

1. Sexo: $\square \quad$ Masculino $\square \quad$ Femenino

2. Edad:___años

3. ¿Tiene usted hijos/as? Sí $\square \quad$ No

4. Estado civil: $\quad$ Soltero $\square \quad$ Casado/En pareja $\square \quad$ Separado/Divorciado/Viudo

5. Actualmente trabaja en: $\quad$ Atención Primaria $\square \quad$ Atención Especializada

6. Experiencia profesional:____años/___ meses

7. Plan de estudios realizados: Ayudante Técnico Sanitario (A.T.S.)

Diplomatura en Enfermería (D.U.E.)

Grado en Enfermería

8. Universidad y campus de procedencia: Universidad de Murcia (Cartagena) Universidad de Murcia (Espinardo) Universidad de Murcia (Lorca) Otra (Indique cual):

9. ¿Cursó la asignatura de Cuidados Paliativos en su plan de estudios?

Sí No

10. ¿Tiene formación específica en Cuidados Paliativos? *Si la respuesta es NO, pase a la pregunta 13

11. ¿Dónde recibió su formación en Cuidados Paliativos? (Puede seleccionar varias opciones) Grado/Diplomatura Formación continuada/Cursos

Experto Universitario

Máster

12. Horas de formación en Cuidados Paliativos: Menos de 80 horas

80-150 horas

Más de 150 horas

13. ¿Tiene experiencia profesional en Cuidados Paliativos?

Sí

No

14. ¿Cuánto tiempo? años/ meses 
15. ¿Está usted en contacto actualmente en su trabajo con pacientes que necesitan Cuidados Paliativos? Sí $\square \quad$ No, pero lo he estado $\square \quad$ No, nunca lo he estado

16. El Plan Integral de Cuidados Paliativos de la Región de Murcia: (Puede seleccionar varias opciones) Lo conozco $\square \quad$ Lo utilizo $\quad \square \quad$ No lo conozco $\quad \square \quad$ No lo utilizo

17. La formación que recibe el profesional de Enfermería en Cuidados Paliativos es para usted: Suficiente $\square \quad$ Insuficiente

19. Se considera capacitado para proporcionar cuidados a pacientes terminales y a sus familias? Sí $\square \quad$ No $\square$

CUESTIONARIO SOBRE LOS CONOCIMIENTOS DE ENFERMERÍA DE LOS CUIDADOS PALIATIVOS (PCQN) Por favor, señale con una " $X$ " su respuesta en estas 20 preguntas relacionadas con conceptos básicos sobre Cuidados Paliativos:

\begin{tabular}{|c|c|c|c|c|}
\hline & & Verdadero & Falso & No lo sé \\
\hline 1 & $\begin{array}{l}\text { Los Cuidados Paliativos son apropiados solo en situaciones en la que hay evidencia } \\
\text { de empeoramiento o deterioro de la situación clínica. }\end{array}$ & & & \\
\hline 2 & $\begin{array}{l}\text { La morfina es el estándar utilizado para comparar el efecto analgésico de otros } \\
\text { opioides. }\end{array}$ & & & \\
\hline 3 & $\begin{array}{l}\text { La extensión de la enfermedad determina la vía de administración de tratamiento } \\
\text { para el dolor. }\end{array}$ & & & \\
\hline 4 & Las terapias adyuvantes son importantes en el manejo del dolor. & & & \\
\hline 5 & $\begin{array}{l}\text { Es esencial para todas las familias permanecer al lado del enfermo hasta su } \\
\text { fallecimiento. }\end{array}$ & & & \\
\hline 6 & $\begin{array}{l}\text { Durante los últimos días de vida, la somnolencia asociada al desequilibrio } \\
\text { electrolítico puede disminuir la necesidad de sedación. }\end{array}$ & & & \\
\hline 7 & $\begin{array}{l}\text { La adicción es un gran problema cuando se usa morfina como tratamiento de base } \\
\text { para el manejo del dolor a largo plazo. }\end{array}$ & & & \\
\hline 8 & $\begin{array}{l}\text { Los individuos que toman opioides deberían seguir medidas para mejorar la } \\
\text { evacuación intestinal. }\end{array}$ & & & \\
\hline 9 & $\begin{array}{l}\text { Para proporcionar cuidados paliativos se necesita establecer un distanciamiento } \\
\text { emocional. }\end{array}$ & & & \\
\hline 10 & $\begin{array}{l}\text { Durante las fases finales de una enfermedad, los fármacos que pueden causar } \\
\text { depresión respiratoria son apropiados para tratar la disnea severa. }\end{array}$ & & & \\
\hline 11 & Los hombres, generalmente, resuelven su duelo más rápido que las mujeres. & & & \\
\hline 12 & La filosofía de los cuidados paliativos es compatible con tratamientos activos. & & & \\
\hline 13 & El uso de placebos es apropiado en el tratamiento de algunos tipos de dolor. & & & \\
\hline 14 & A dosis altas, la codeína causa más náuseas y vómitos que la morfina. & & & \\
\hline 15 & Sufrimiento y dolor físico son sinónimos. & & & \\
\hline 16 & La dolantina no es un analgésico efectivo en el control del dolor crónico. & & & \\
\hline 17 & $\begin{array}{l}\text { La acumulación de pérdidas hace que el burnout sea inevitable para aquellos } \\
\text { que trabajan en cuidados paliativos. }\end{array}$ & & & \\
\hline 18 & Las manifestaciones del dolor crónico son diferentes de las del dolor agudo. & & & \\
\hline 19 & $\begin{array}{l}\text { La pérdida de alguien con quien se tiene una relación distante o conflictiva es más } \\
\text { fácil de resolver que la de alguien con quien se tiene una relación más cercana o } \\
\text { Íntima. }\end{array}$ & & & \\
\hline 20 & El umbral del dolor se ve reducido por la ansiedad o la fatiga. & & & \\
\hline
\end{tabular}




\section{Bibliografía}

1. Martínez MB, Monleón M, Carretero Y, García-Barquero MT. Enfermería en Cuidados Paliativos y al final de la vida. $1 .^{a}$ ed. Barcelona: Elsevier España; 2012.

2. Bermejo JC, Díaz-Albo E, Sánchez E. Manual básico para la atención integral en Cuidados Paliativos. 1. ${ }^{\mathrm{a}}$ ed. Madrid: Cáritas; 2011.

3. López E. Enfermería en Cuidados Paliativos. 1. ${ }^{a}$ ed. Madrid: Editorial Médica Panamericana; 2003.

4. García A. Enfermería en Cuidados Paliativos. 1. ${ }^{a}$ ed. Madrid: Editorial Universitaria Ramón Areces; 2011.

5. Codorniu N, Guanter L, Molins A, Utor L. Competencias enfermeras en Cuidados Paliativos. Madrid: Sociedad Española de Cuidados Paliativos (SECPAL); 2013 [Último acceso: 5 de noviembre de 2019]. Disponible en: http://www.secpal.com/\%5CDoc umentos\%5CBlog\%5CMONOGRAFIA\%203.pdf

6. Hernández ML, Aguilar CR. Conocimientos del personal de enfermería sobre Cuidados Paliativos en pacientes hospitalizados de Medicina Interna. Rev Enferm IMSS. 2016;24:87-90.

7. Kada O, Janig H, Pinter G, Cernic K, Likar R. Palliative Care in nursing homes: Results of a survey about knowledge and selfefficacy of nursing staff. Schmerz. 2017;31:383-90.

8. Unroe KT, Cagle JG, Lane KA, Callahan CM, Miller SC. Nursing Home Staff Palliative Care Knowledge and Practices: Results of a Large Survey of Frontline Workers. J Pain Symtom Manage. 2015;50:622-9.

9. Autor SH, Storey SL, Ziemba-Davis M. Knowledge of Palliative Care. An Evaluation of Oncology, Intensive Care, and Heart Failure Nurses. J Hosp Palliat Nurs. 2013;15:307-14.

10. Chover E, Martínez A, Lapeña YR. Knowledge in Palliative Care of nursing professionals at a Spanish hospital. Rev. Latino-Am. Enfermagem. 2017;25:1-8.

11. Cuevas CS. Nivel de conocimiento en Cuidados Paliativos de los profesionales de Atención Primaria. Identificación de los factores asociados [Trabajo Fin de Máster]. Universidad de Murcia; 2013 [Último acceso: 5 de noviembre de 2019]. Disponible en: https: //goo.gl/Z68Me4

12. Chover E, Martínez A, Lapeña YR. An instrument to measure nurses' knowledge in Palliative Care: Validation of the Spanish version of Palliative Care Quiz for Nurses. PLoS One. 2017;12:1-15.

13. Slåtten K, Hatlevik O, Fagerström L. Validation of a New Instrument for Self-Assessment of Nurses' Core Competencies in Palliative Care. Nurs Res Pract. 2014;2014:615498.

14. Prem V, Karvannan H, Kumar SP, Karthikbabu S, Syed N, Sisodia V, et al. Study of Nurses' Knowledge about Palliative Care: A Quantitative Cross-sectional Survey. Indian J Palliat Care. 2012;18:122-7.
15. Kassa H, Murugan R, Zewdu F, Hailu M, Woldeyohannes D. Assessment of knowledge, attitude and practice and associated factors towards Palliative Care among nurses working in selected hospitals, Addis Ababa, Ethiopia. BMC Palliat Care. 2014;13:1-11.

16. Iranmanesh S, Razban F, Tirgari B, Zahra G. Nurses' knowledge about and attitude towards Palliative Care in Southeast Iran. Palliat Support Care. 2014;12:203-10.

17. Katumbo A, Mwinkeu N, Kabamba M, Mundongo H, Ntambue A, Ilunga J, et al. Assessment of nurses' knowledge, attitude and associated factors towards Palliative Care in Lubumbashi's Hospitals. Int J Sci Res. 2015;6:922-8.

18. Choi M, Lee J, Kim SS, Kim D, Kim H. Nurses' Knowledge about end-of-life care: Where are we? J Contin Educ Nurs. 2012;43:379-84.

19. Al Qadire M. Nurses' Knowledge about Palliative Care: A crosssectional survey. J Hosp Palliat Nurs. 2014;16:1-8.

20. Karikwera MC. Assessment of nurses' knowledge and attitude about Palliative Care among nurses at University teaching hospital of Kigali [Trabajo Fin de Máster]. University of Rwanda; 2017 [Último acceso: 5 de noviembre de 2019]. Disponible en: https: //goo.gl/vkpctT

21. Boon K, Hyun K, Su Yu, Sung C, Yun J, So-Hi K. Evaluation of End-of-Life Nursing Education Consortium-Geriatric Train-theTrainer Program in Korea. Korean J Adult Nurs. 2012;24:390-7.

22. Brazil K, Brink P, Kaasalainen S, Kelly ML, McAiney C. Knowledge and perceived competence among nurses caring for the dying in long-term care homes. Int J Palliat Nurs. 2012;18:77-83.

23. Chover E, Martínez A. Utility of social networks and online data collection in nursing research: Analysis of Spanish nurses' level of knowledge about Palliative Care. PLoS ONE. 2018;13:1-16.

24. Hyun K, Boon K, Su Yu, Sanghee K, Sook P, Sungeun C, et al. The effect of and End-ofLife Nursing Education Consortium Course on Nurses' knowledge of Hospice and Palliative Care in Korea. J Hospice Palliat Nurs. 2011;13:222-9.

25. Ross M, McDonald B, McGuiness J. The Palliative Care Quiz for Nursing (PCQN): The development of an instrument to measure nurses' knowledge of Palliative Care. J Adv Nurs. 1996;23:126-37.

26. Taleghani F, Shahriari M, Alimohammadi N. Empowering Nurses in providing Palliative Care to cancer patients: Action research study. Indian J Palliat Care. 2018;24:98-103.

27. Satsin T, Matchim Y, Thongthawee B. Emergency Nurses' competency in the provision of Palliative Care and related factors. Songklanagarind J Nurs. 2017;37:41-50. 